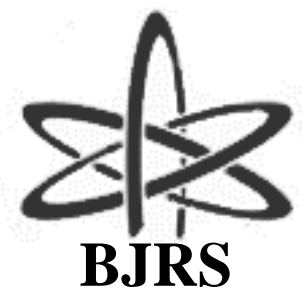

BRAZILIAN JOURNAL

$\mathrm{OF}$

RADIATION SCIENCES

09-01 (2021) 01-13

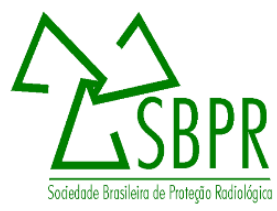

\title{
Dose periférica em radioterapia: comparação entre diferentes equipamentos aceleradores lineares e mesmo equipamento com diferentes energias de fótons
}

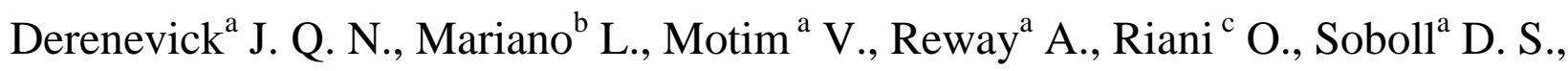 \\ Malthez ${ }^{a}$ A. L. M. C. \\ (a) Universidade Tecnológica Federal do Paraná (UTFPR) \\ Av. Sete de Setembro 3165, Rebouças - 80230-901 Curitiba, PR - Brasil \\ (b) Universidade de São Paulo (USP) \\ Rua do Matão, 1371, Cidade Universitária - 05508-090 São Paulo - SP \\ ${ }^{(c)}$ Clínica Oncoville \\ Rodovia Curitiba - Ponta Grossa Br-277, 1437 - Mossunguê - 82305-100 Curitiba - PR - Brasil
}

josiane_querino@yahoo.com.br

RESUMO

A dose de radiação fora do campo de tratamento de radioterapia pode ser uma preocupação clínica. Portanto, um método para predizer com precisão as doses periféricas recebidas pelos tecidos e a avaliação adequada das doses é necessário para a proteção radiológica de pacientes em tratamentos de radioterapia. $O$ objetivo deste trabalho foi avaliar a dose recebida em áreas periféricas fora da área alvo para tratamentos com feixes de fótons de $6 \mathrm{MV}$ e $15 \mathrm{MV}$ de dois aceleradores lineares diferentes, usando um simulador antropomórfico e, assim, comparar os valores obtidos entre os dois equipamentos e entre diferentes energias no mesmo equipamento. Embora os equipamentos e cuidados durante o planejamento busquem minimizar as doses fora da região de tratamento, observamos experimentalmente que mesmo usando tamanho de campo relativamente pequeno e a mesma dose, diferenças nas doses periféricas são observadas, considerando fótons da mesma energia de diferentes equipamentos aceleradores e fótons com energias diferentes empregadas no mesmo equipamento acelerador linear.

Palavras-chave: Dose periférica, Acelerador Linear, Tratamentos Radioterápicos. 


\begin{abstract}
The radiation dose outside the radiotherapy treatment field can be a clinical concern. Therefore, a method of accurately predicting the peripheral doses received by tissues and appropriated evaluation of doses are required for radiation protection of patients in radiotherapy treatments. The objective of this work was to measure dose received at peripheral areas outside of target area for incident photon beams of $6 \mathrm{MV}$ and $15 \mathrm{MV}$ from two different linear accelerators using an anthropomorphic phantom and thus compare the values obtained between the two equipment and between different energies in the same equipment. Although equipment and care during planning seek to minimize doses outside the treatment region, we have experimentally observed that, even using relatively small field size and the same dose, differences in peripheral doses are observed, considering photons of the same energy from different accelerator equipment and photons with different energies from the same linear accelerator equipment.
\end{abstract}

Keywords: Peripheral dose, Linear Accelerator, Radiotherapy Treatments.

\title{
1. INTRODUÇÃO
}

No Brasil, estima-se cerca de 309 mil para homens e 316 mil para mulheres novos casos de câncer nos anos entre 2020 e 2022 [1]. A radioterapia é uma modalidade terapêutica que emprega radiações ionizantes com objetivo de destruir as células tumorais e/ou impedir o crescimento desordenado dessas células. Atualmente, os equipamentos mais utilizados para radioterapia são os aceleradores lineares que podem empregar tanto feixe de fótons como elétrons na faixa de megavoltagem para realização de tratamentos oncológicos.

Em todo planejamento radioterápico, os tecidos sadios que estão ao redor do tumor recebem radiação. Essas doses podem acarretar danos tardios, prejudicando a qualidade de vida do paciente. A finalidade da dosimetria periférica é quantificar a dose absorvida fora do campo de radiação terapêutico [2]. A dose periférica é composta basicamente, por três situações: radiação espalhada pelo alvo, pela sala de tratamento e em virtude da radiação de fuga do cabeçote [3]. Na primeira, a dose periférica incide no alvo de tratamento e é espalhada pelo meio. Na segunda, a radiação espalhada pela sala de tratamento depende das dimensões e dos materiais presentes, assim como da energia e do equipamento utilizado. Dessa forma, cada sala possui fatores específicos que devem 
ser analisados de maneira individual. A radiação que não pertence ao feixe útil, mas que é extravasada pelo cabeçote do equipamento ou pelo sistema de colimação, caracteriza a terceira situação responsável pela composição da radiação periférica [3].

Desde a década de 1970, tem havido um crescente interesse nas doses de radiação fora do campo de irradiação para o tratamento radioterápico. O foco dos estudos é tipicamente uma dose de uma perspectiva de segurança de radiação ocupacional ou doses para estruturas críticas não direcionadas no corpo do paciente. De modo geral, analisando os diversos estudos publicados, as doses, no caso de fótons, fora do volume de tratamento, diminuem com o tamanho do campo e caem aproximadamente exponencialmente conforme se afasta da borda de campo [4].

Embora o planejamento em radioterapia minimize as doses recebidas por estruturas adjacentes e radiossensíveis fora da região que se deseja tratar, é relatado na literatura que as doses periféricas recebidas durante a aplicação do tratamento dependem das configurações da máquina e da técnica utilizada, além da própria anatomia do paciente $[5,6]$.

Considerando a proteção radiológica dos pacientes oncológicos e a minimização dos efeitos biológicos da radiação ionizante, este trabalho teve como objetivo quantificar a dose periférica em diferentes equipamentos de radioterapia com fótons de mesma energia e no mesmo equipamento considerando feixes de fótons com energias diferentes.

\section{MATERIAIS E MÉTODOS}

Para avaliar as doses periféricas considerando diferentes equipamentos e o mesmo equipamento com diferentes energias, foram realizadas irradiações na clínica de radioterapia Oncoville, localizada em Curitiba/PR, em dois aceleradores lineares da marca Varian; um modelo CL2100 com feixes de fótons de $6 \mathrm{MV}$ e $15 \mathrm{MV}$ e um segundo modelo CL600CD com fótons de $6 \mathrm{MV}$.

As doses periféricas foram avaliadas utilizando detectores TL de fluoreto de lítio dopado com magnésio e titânio ( $\mathrm{LiF}: \mathrm{Mg}$, Ti), com 3,1 mm de lado e 0,9 mm de espessura, disponíveis comercialmente com o nome de TLD100 (Bicron Co), os quais foram previamente selecionados por sensibilidade $(<6 \%)$ e calibrados para cada equipamento e energia. 
A dose periférica foi obtida irradiando um simulador antropomórfico adulto (Alderson Radiation Therapy Phanton - ART-210), disponibilizado pela UTFPR, é composto por fatias com densidade equivalente do corpo humano, onde cada fatia possui orifícios que permitem a inserção de detectores para realização de medidas em profundidades.

Desta forma, os detectores TLD100, embalados aos pares em plástico preto radiotransparente, foram inseridos ou posicionados nos cristalinos direito e esquerdo, tireoide, mama esquerda, abdomen e gônadas. Além da sensibilidade à radiação, essas regiões foram escolhidas por estarem em posições diferentes com relação ao isocentro, permitindo assim a avaliação da dose periférica em diferentes distâncias, e considerando também diferentes profundidades no objeto simulador.

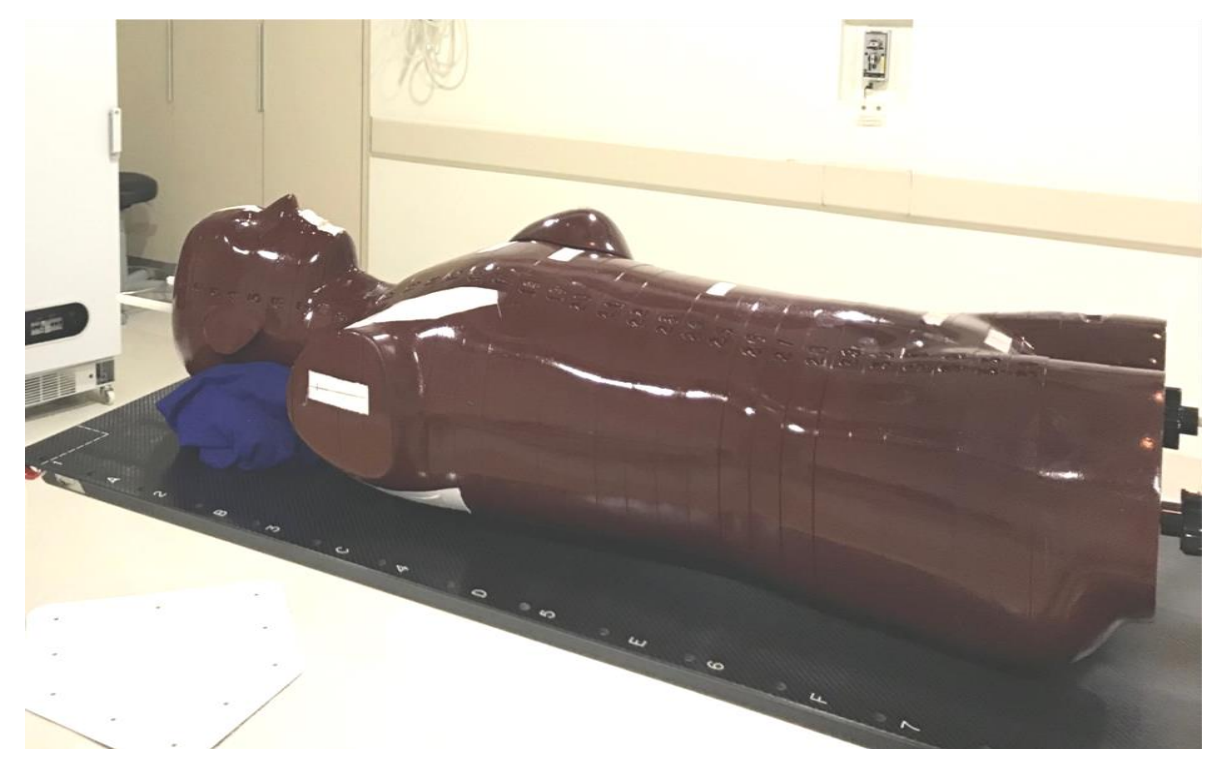

Figura 1: Simulador antropomórfico adulto.

No planejamento, o isocentro foi posicionado na região da cabeça entre as fatias 1 e 2 do objeto simulador e irradiado nos dois equipamentos aceleradores com $20 \mathrm{~Gy}$, empregando feixes de fótons de $6 \mathrm{MV}$ dos diferentes equipamentos e os feixes de 6 e $15 \mathrm{MV}$ do mesmo equipamento acelerador. A dose de $20 \mathrm{~Gy}$, relativamente alta, foi escolhida considerando protocolos em radioterapia com dose única e o menor tamanho de campo possível considerando o posicionamento do simulador no equipamento. 


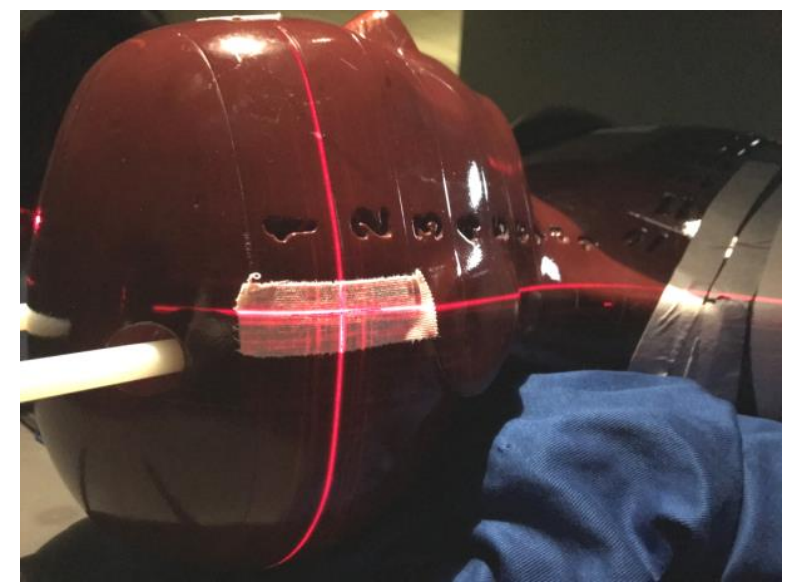

Figura 2: Posicionamento do isocentro.

Em todas as irradiações foi empregado o mesmo tamanho de campo $\left(5 \times 5 \mathrm{~cm}^{2}\right)$ e a mesma dose foi entregue no isocentro $(20 \mathrm{~Gy})$, para avaliar diferenças na dose periférica entre as diferentes energias e estrutura aceleradora dos equipamentos. Para isso, com o feixe de fótons de $6 \mathrm{MV}$ no equipamento CL2100, foram empregadas 2810 UM e uma distância da fonte - superfície de 90,5 cm. No feixe de 15 MV, no modelo CL2100, foram ajustadas 2346 UM e uma distância da fonte superfície de 90,5 cm. Para o terceiro feixe de 6MV, no modelo CL600CD, foram empregadas 2808 UM com uma distância da fonte - superfície de $90,5 \mathrm{~cm}$. Para cada energia, foi realizada somente uma exposição, ou seja, aplicado somente uma vez o tratamento radioterápico planejado no isocentro.

As leituras dos detectores, tanto utilizados na calibração como posicionados nas diferentes regiões anatômicas do simulador, foram realizadas dois dias após as irradiações. Os detectores foram lidos em um leitor automatizado Riso TL/OSL DA20 utilizando filtro Roya U340 posicionado na frente do tubo fotomultiplicador alimentado com $1225 \mathrm{~V}$ para detecção do sinal luminoso. Para estimulação, os TLD100 foram aquecidos até a temperatura de $350^{\circ} \mathrm{C}$ utilizando uma taxa de aquecimento de $5^{\circ} \mathrm{C} / \mathrm{s}$.

As intensidades TL obtidas na leitura foram convertidas em dose absorvida por meio de fatores de calibração previamente avaliados para cada energia de fótons empregada. Para isso, três conjuntos de detectores TL foram irradiados em cada situação de irradiação com quatro diferentes valores de doses (100, 200, 250 e 300 cGy) em cada feixe, entre placas de água sólida para condição de equilíbrio eletrônico. Em seguida, os fatores de calibração foram avaliados por meio do 
ajuste de uma função de primeiro grau aos valores de intensidade TL em função da dose. O coeficiente angular do ajuste fornece a sensibilidade (contagens por cGy) do TLD100 para a energia do feixe, desse modo, dividindo o valor de intensidade TL pela sensibilidade é obtida a dose a qual o detector foi exposto.

A avaliação das incertezas, do tipo A, foi realizada considerando-se a incerteza na sensibilidade obtida no ajuste linear da intensidade TL em função da dose e o desvio padrão dos valores de intensidade TL obtido no processo de seleção dos dosímetros. Os valores de dose periférica obtidos nas irradiações com os diferentes equipamentos e energias foram comparados utilizando o teste $\mathrm{Z}$ $[7]$.

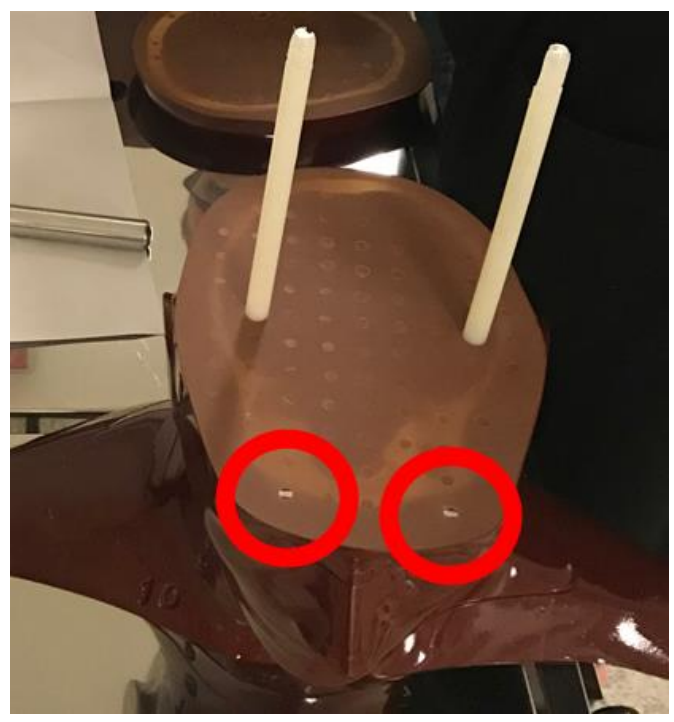

Figura 3: TLD posicionados em região do cristalino.

\section{RESULTADOS E DISCUSSÃO}

A Figura 4 mostra os ajustes lineares dos valores de intensidade TL em função da dose para os feixes de fótons de 6 e 15 MV dos dois equipamentos aceleradores lineares (Varian CL2100 e CL600) utilizados nesse estudo.

Os valores de intensidade TL dos detectores posicionados no simulador antropomórfico foram convertidos em dose utilizando os valores de sensibilidade avaliados por meio do Método dos 
Mínimos Quadrados para fazer o ajuste linear mostrado na Figura 4. Para o feixe de fótons de 6 e 15 MV do equipamento Varian CL2100 o TLD100 apresentou sensibilidades de $(4,4 \pm 0,3) \cdot 10^{4}$ ctgs/cGy e $(5,1 \pm 0,4) \cdot 10^{4} \mathrm{ctgs} / \mathrm{cGy}$ e $(5,0 \pm 0,4) \cdot 10^{4} \mathrm{ctgs} / \mathrm{cGy}$ para a energia do feixe de fótons de 6 MV do acelerador Varian CL600.

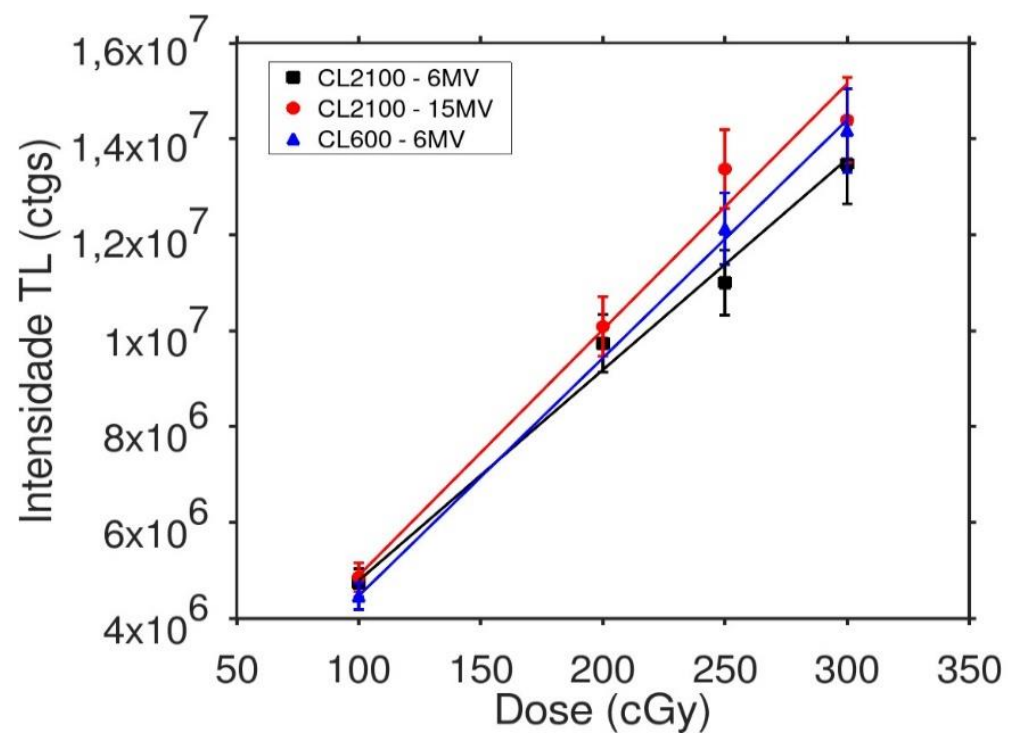

Figura 4: Ajuste linear dos valores de intensidade TL em função da dose obtidos por meio dos detectores TLD100 expostos a fótons de 6 e 15 MV em um acelerador linear Varian CL2100 e a fótons de $6 \mathrm{MV}$ em um acelerador linear Varian $6 \mathrm{MV}$.

A Tabela 1 apresenta os valores de dose periférica avaliados nas diferentes regiões do objeto simulador, quando empregados feixes de fótons com a mesma energia (fótons de 6 MV) provenientes de diferentes aceleradores (CL2100 e CL600) e empregando fótons com energias diferentes (fótons de 6 e $15 \mathrm{MV}$ ) provenientes do mesmo equipamento acelerador (CL2100). 
Tabela 1: Dose periférica e sua respectiva incerteza para diferentes equipamentos considerando a mesma energia e para o mesmo equipamento considerando energias distintas.

\begin{tabular}{lccc}
\hline & \multicolumn{3}{c}{ Dose (cGy) } \\
\cline { 2 - 4 } & \multicolumn{2}{c}{ Varian CL2100 } & Varian CL600 \\
\hline Região & $\mathbf{6 ~ M V}$ & $\mathbf{1 5} \mathbf{~ M V}$ & $\mathbf{6 ~ M V}$ \\
\hline Cristalino direito & $35 \pm 3$ & $46 \pm 4$ & $36 \pm 3$ \\
Cristalino esquerdo & $38 \pm 4$ & $36 \pm 3$ & $36 \pm 3$ \\
Tireoide & $6,6 \pm 0,6$ & $8,6 \pm 0,8$ & $8,3 \pm 0,8$ \\
Mama & $0,62 \pm 0,07$ & $2,5 \pm 0,2$ & $0,54 \pm 0,06$ \\
Abdômen & $0,33 \pm 0,04$ & $2,2 \pm 0,2$ & $0,12 \pm 0,02$ \\
Gônadas & $0,20 \pm 0,03$ & $1,9 \pm 0,2$ & $0,20 \pm 0,02$ \\
\hline
\end{tabular}

As doses medidas pelos dosímetros TL posicionados em região dos cristalinos direito e esquerdo, tireoide, mama, abdomen e gônadas variaram entre 46 a 0,12 cGy. Como já esperado, os cristalinos são os locais de maior recebimento de dose dentre as estruturas avaliadas, devido à sua proximidade da região anatômica com o isocentro.

A Tabela 2 apresenta o resultado do teste $\mathrm{Z}$ comparando os resultados das doses periféricas avaliadas em todas as situações de irradiações realizadas.

Tabela 2: Resultado do teste $\mathrm{Z}$ para comparação dos diferentes equipamentos considerando energias distintas e diferentes equipamentos considerando a mesma energia e energias distintas.

\begin{tabular}{lccc}
\hline Região & $\begin{array}{c}\text { Fótons de 6 MV e } \\
\mathbf{1 5} \text { MV (CL2100) }\end{array}$ & $\begin{array}{c}\text { Fótons de } \\
\text { 6 MV } \\
\text { (CL2100 e CL600) }\end{array}$ & $\begin{array}{c}\text { Fótons de } \\
\mathbf{1 5} \text { MV (CL2100) e } \\
\text { 6 MV (CL600) }\end{array}$ \\
\hline Cristalino direito & 2,0 & 0,1 & 1,9 \\
Cristalino esquerdo & 0,4 & 0,5 & 0,1 \\
Tireoide & 2,0 & 1,7 & 0,3 \\
Mama & 7,6 & 0,9 & 8,0 \\
Abdômen & 8,7 & 5,1 & 9,8 \\
Gônadas & 9,2 & 0,2 & 9,2 \\
\hline
\end{tabular}

No teste Z, valores abaixo de 3 foram considerados compatíveis do ponto de vista estatístico e incompatíveis quando acima de 3. Deste modo, comparando a dose periférica avaliada para as irradiações em diferentes equipamentos empregando a mesma energia (segunda coluna da Tabela 
2), não foram observadas diferenças estatísticas significativas, exceto para região do abdome, na qual a dose absorvida foi aproximadamente 3 vezes maior para irradiação no equipamento CL2100, quando comparada com a irradiação no equipamento CL600. Considerando o mesmo equipamento, porém em feixes de energias distintas (primeira coluna da Tabela 2), observaram-se valores de dose periférica maiores para energia mais alta nas regiões anatômicas distantes do isocentro (6 e $15 \mathrm{MV}$ ).

Além disso, observou-se também que quanto mais distante do isocentro menor foi a dose periférica. Comparando os valores obtidos da dose absorvida na região do cristalino com a dose absorvida na região das gônadas, essas são de aproximadamente $4 \%$ da dose recebida nas regiões próximas do isocentro no caso do feixe de $15 \mathrm{MV}$.

Estudos publicados mostram que, embora não haja grande variação na dose de radiação de fuga entre marcas diferentes de aceleradores, algumas apresentam maior dose de dispersão de colimador do que outras. Porém com os valores de dose dentro dos limites regulamentares [8].

Fraass e Geijn [6] investigaram a dose periférica para um feixe $\mathrm{de}^{60} \mathrm{Co}$, assim como feixes de fótons de 4, 6 e $8 \mathrm{MV}$. As doses foram relatadas para medições no objeto simulador de água para vários tamanhos de campo em uma faixa de distâncias da borda do campo e a dosimetria termoluminescente (TL) foi empregada para medidas de dose no tratamento de pacientes. Kase et al [9] estudaram similarmente um feixe de ${ }^{60} \mathrm{Co}$, assim como feixes de fótons de 4 e $8 \mathrm{MV}$. Os autores também diferenciaram a radiação de fuga do cabeçote e a radiação espalhada, descobrindo que o espalhamento do colimador pode contribuir com cerca de $40 \%$ da dose fora do campo de tratamento. Francois et al [10] parametrizaram distribuições de dose para diferentes energias de feixe em função da profundidade, distância da borda, tamanho do campo e forma. Um algoritmo foi desenvolvido para determinar a dose para órgãos fora do feixe para distâncias de 10 a $50 \mathrm{~cm}$ da borda do campo. As medições foram realizadas com detectores termoluminescentes (TLD) em um objeto simulador antropomórfico para vários tamanhos de campo, adicionalmente também foram realizadas simulações utilizando o Método de Monte Carlo.

Van der Giessen [8] mediu as doses periféricas em um simulador de água para 4 equipamentos com fontes de ${ }^{60} \mathrm{Co}$ e 37 aceleradores lineares para investigar a variação em doses periféricas entre máquinas de 7 fabricantes diferentes. A parcela da dose correspondente ao espalhamento foi pequena entre os modelos variados, no entanto, a dose de colimador encontrada foi de até $50 \%$, dependendo do projeto do colimador/filtro de achatamento. 
Em sua tese de doutorado, Van der Giessen [11] fornece resultados de estudos de várias máquinas (com foco em equipamentos com fonte de ${ }^{60} \mathrm{Co}$ ), principalmente usando objeto simulador de água para coletar dados ou por avaliação de dados publicados e dados de dispersão no colimador/ espalhamentos fornecidos por clínicas e instituições. Neste trabalho, a dose também foi medida no períneo dos pacientes usando TLD. Os estudos que constituem sua tese foram publicados separadamente como artigos, principalmente no Int. J. Radiat. Oncol. Biol. Phys. [10, 12-15].

Os valores obtidos neste trabalho estão em concordância com o estudo de Van der Giessen et al, em que foram realizadas as medições de dose periférica no paciente durante situação real de tratamento, através de TLD em região de períneo. Em todas as medidas juntas, os valores calculados excederam as doses periféricas medidas em cerca de 9\%, com um desvio padrão de $35 \%$. A correlação variou entre subgrupos específicos, mas a diferença entre a medição e o cálculo não excedeu $50 \%$ [15].

Estudos já publicados mostram que como a dose fora do campo para regiões não segmentadas do corpo de um paciente é o resultado de uma combinação de radiação de fuga e dispersão, é provável que modelos diferentes de aceleradores lineares (com diferentes designs de blindagem) gerem diferentes doses fora do campo [4]. Sendo assim, equipamentos de alta energia podem apresentar dose periférica maior quando comparado com equipamentos de menor energia, pois em aceleradores lineares com emissão de fótons acima de $6 \mathrm{MV}$ possuem o tubo acelerador na posição horizontal, devido ao seu maior comprimento e bending magnet, enquanto aceleradores de $4 \mathrm{MV}$ e alguns modelos de $6 \mathrm{MV}$ possuem o tubo acelerador na posição vertical por possuírem um comprimento menor, e devido sua posição na vertical já estar direcionada para a saída do feixe, não há o bending magnet.

No caso dos aceleradores lineares, por exemplo, dados mostram que, mesmo quando operados no mesmo modo de energia (6 MV), há uma diferença significativa entre as doses fora do campo do Varian (multimodo) 2100 e o Varian (monomodo) 600C. A dose avaliada nas irradiações com o Varian 2100 é até $250 \%$ maior para regiões fora do campo do que a avaliada nas irradiações com Varian 600C. Esta discrepância é provavelmente devido ao maior tubo acelerador na horizontal do Varian 2100 e à presença de um bending magnet (este último agindo como uma fonte adicional de bremsstrahlung). O Varian $600 \mathrm{C}$ possui um tubo acelerador na vertical direcionado para o isocentro e sem bending magnet [4]. 
Na tabela 1 é observado que os valores de dose periférica para os dois modelos de aceleradores usados neste estudo são relativamente próximos, apresentando valores de medidas compatíveis para pontos próximos da região do isocentro, como no cristalino direito e esquerdo, sendo de $2 \%$ da dose no isocentro. No caso da mama, comparando a mesma energia, os valores são semelhantes, com $0,03 \%$ da dose no isocentro. No caso da tireoide os valores de medida são compatíveis nos dois equipamentos, sendo a dose periférica entre $0,3 \%$ e $0,4 \%$ da dose no isocentro. Nos demais pontos de medida os valores de dose relativa à dose total no isocentro foram maiores no CL2100.

A maioria dos valores medidos foram maiores no CL2100 e estão em concordância com demais trabalhos que realizaram medidas de dose periférica em condições semelhantes às que foram usadas neste trabalho $[8 ; 12-15]$.

Em comparação com o trabalho dos autores Taylor e Kron [4], no qual foram observadas diferenças da dose espalhada em feixes de $6 \mathrm{MV}$ entre dois equipamentos (Varian 2100 e Varian 600) de até $250 \%$, neste trabalho a maior diferença da dose espalhada para a mesma energia foi na região do abdome de $275 \%$ (aproximadamente 3 vezes maior no equipamento CL2100). Os aceleradores utilizados neste trabalho possuem configurações semelhantes aos equipamentos descritos por esses autores, pois o CL2100 tem o tubo acelerador na horizontal com bending magnet e o CL600 tem o tubo acelerador na vertical e sem bending magnet.

\section{CONCLUSÕES}

Embora os equipamentos e cuidados durante o planejamento busquem minimizar as doses fora da região de tratamento, observamos experimentalmente que mesmo utilizando tamanho de campo relativamente pequeno e uma mesma dose, são observadas diferenças nas doses periféricas quando considerados fótons de mesma energia provenientes de equipamentos aceleradores diferentes e fótons com energias distintas provenientes do mesmo equipamento acelerador linear.

Todos os valores de dose periférica, para uma mesma região anatômica, encontrados neste trabalho são compatíveis entre os dois equipamentos para energias de fótons de $6 \mathrm{MV}$ (exceto na região do abdômen que a dose apresentou uma diferença maior para o CL2100). Considerando diferentes energias tanto no mesmo equipamento quanto em equipamento diferentes, os valores de 
dose periférica variam em pontos distantes do isocentro, sendo as doses periféricas maiores para a maior energia. Estes resultados são semelhantes a trabalhos já publicados na literatura e os valores obtidos estão de acordo com à TG 36.

\section{REFERÊNCIAS}

[1] INCA - Instituto Nacional do Câncer José de Alencar. Disponível em https://www.inca.gov.br. Acesso em setembro de 2020.

[2] BETTA, E. FARISELLI, L. BERGANTIN, A. LOCATELLI, F. VECCHIO, A.D BROGGI, S. FUMAGALLI, M.L. Evaluation of the peripheral dose in stereotactic radiotherapy and radiosurgery treatments. Medical Physics, V 37, n 7, Pg 3587 - 3594. 2010.

[3] VIGNA FILHO, EUGENIO DEL. FALCÃO, ROSSANA C.. Blindagem em Radioterapia: Técnica e Normas. Rio de Janeiro: Inca, 2000.

[4] TAYLOR M. L. AND KRON T. Consideration of the radiation dose delivered away from the treatment field to patients in radiotherapy. J Med Phys. 2011;36(2):59-71.

[5] MUTIC, S., KLEIN, E.E. A reduction in the AAPM TG-36 reported peripheral dose distributions with tertiary multileaf collimation. American Association of Physicists in Medicine Task Group 36. Int J Radiat Oncol Biol Phys. 1999; 44(4):947-53.

[6] FRAASS B, GEIJN JV. Peripheral dose from megavolt beams. Med Phys. 1983;10:809-18.

[7] MAGALHÃES, M N, LIMA, A C P. Noções de Probabilidade e Estatística. $7^{\mathrm{a}}$ Edição, $3^{\mathrm{a}}$ reimpressão revista. São Paulo: Edusp.

[8] VAN DER GIESSEN P. Collimator-related radiation dose for different cobalt machines and linear accelerators. Int J Radiat Oncol Biol Phys. 1996;35:399-405. 
[9] KASE K, SVENSSON G, WOLBARST A, MARKS M. Measurements of dose from secondary radiation outside a treatment field. Int J Radiat Oncol Biol Phys. 1983;9:1173-83.

[10] FRANCOIS P, BEURTHERET C, DUTREIX A. Calculation of the dose delivered to organs outside the radiation beams. Med Phys. 1988; 15: 879-883.

[11] VAN DER GIESSEN P. PhD Thesis Dose outside the irradiated volume in radiotherapy.Gottingen Tilberg: The Netherlands; 1997a.

[12] VAN DER GIESSEN P, HURKMANS C. Calculation and measurement of the dose to points outside the primary beam for Co-60 gamma radiation. Int J Radiat Oncol Biol Phys. 1993; 27:717-24.

[13] VAN DER GIESSEN P. Calculation and measurement of the dose at points outside the primary beam for photon energies of 6,10 and $23 \mathrm{MeV}$. Int J Radiat Oncol Biol Phys. 1994; 30:1239-46.

[14] VAN DER GIESSEN P. Measurement of the peripheral dose for the tangential breast treatment technique with Co-60 gamma radiation and high energy X-rays. Radiother Oncol. 1997b; 42:265-70.

[15] VAN DER GIESSEN P, BIERHUIZEN W. Comparison of measured and calculated peripheral doses in patients undergoing radiation therapy. Radiother Oncol. 1997; 42:257-64. 